\title{
Oral administration of succinoglycan riclin improves diet-induced \\ hypercholesterolemia in mice
}

\section{Supporting information}

Figure S1. Typical $500 \mathrm{MHz}{ }^{1} \mathrm{H}$ NMR spectra for lipophilic (A) and polar metabolites (B) from four groups with metabolites assigned. In figure $\mathrm{A}, 1$ Cholesterol backbone- $\mathrm{C}(18)-\mathrm{H}_{3}, 2$ Cholesterol $\mathrm{C}(26)-\mathrm{H}_{3}, 3$ Cholesterol $\mathrm{C}(27)-\mathrm{H}_{3}, 4$ Cyclic fatty acids- $\mathrm{CH}_{3}, 5$ Cholesterol $\mathrm{C}(21)-$ $\mathrm{H}_{3}, 6$ Cholesterol $\mathrm{C}(19)-\mathrm{H}_{3}, 7 \mathrm{CH}_{3}$ in fatty acyl chain, $8 \beta-\mathrm{CH}_{2}, 9$ Cholesterol $(-\mathrm{CH}=\mathrm{CH}-\mathrm{C}$ $\left.\mathrm{H}_{2}-\right), 10$ Cholesterol acetate, 11 Unsaturated lipids(- $\left.\mathrm{CH}_{2} \mathrm{CH}=\mathrm{CH}-\right), 12 \mathrm{CH}_{2} \mathrm{COOH}$ in fatty acyl chain, 13 Cholesterol $\mathrm{C}(4)-\mathrm{H}_{3}, 14$ Lipids $\left(\mathrm{CH}_{2} \mathrm{C}=\mathrm{O}\right), 15$ Unsaturated lipids $(=\mathrm{CHC}$ $\left.\mathrm{H}_{2} \mathrm{CH}=\right), 16 \mathrm{~N}+\left(\mathrm{CH}_{3}\right)_{3}, 17$ Glyceryl of phospholipids $\left(-\mathrm{CH}_{2}\right), 18$ Glyceryl of lipids(- $\left.-\mathrm{CH}_{2}\right), 19$ Glyceryl of lipids(-CH), 20 Unsaturated lipids(- $\mathrm{CH}=\mathrm{CH}-$ ); In figure $\mathrm{B}, 1$ Lipids, 2 Isoleucine, 3 Leucine, 4 Valine, 5 Lactate, 6 Alanine, 7 Lysine, 8 Acetate, 9 Glutamine, 10 Glutamate, 11 Succinate, 12 Glutathione, 13 Methionine, 14 Taurine, 15 Betaine, 16 Glucose, 17 Maltose, 18 Uridine, 19 UDP-glucose, $20 \mathrm{NAD}^{+}, 21$ Adenosine, 22 Fumarate, 23 Tyrosine, 24 Histamine, 25 Inosine, 26 ADP, 27 ATP, 28 Xanthine, 29 Phenylalanine, 30 Niacinamide.

Figure S2. OSC-PLS-DA analyses of lipophilic profiles among ND, HCD and HR groups. Score plots and color-coded coefficient loadings plots (A) between ND and HCD groups. Score plots and color-coded coefficient loadings plots (B) between ND and HR groups. Symbols of $\bullet($ red filled squares), $\bullet$ (black filled circles), and $\bullet$ (blue filled rhombuses) represent the ND, HCD, and HR groups, respectively.

Figure S3. OSC-PLS-DA analysis of metabolites data from ND, HCD and HR groups. Score plots (A), S-plot (C) and color-coded coefficient loadings plots (B and D) between ND and HCD groups. Score plots (E), S-plot (G) and color-coded coefficient loadings plots (F and H) between ND and HR groups. Symbols of • (red filled squares), •(black filled circles), and • (blue filled rhombuses) represent the ND, HCD, and HR groups, respectively.

Figure S4. Effects of riclin on the protein $(A, N=3)$ and mRNA levels $(B, N=5-6)$ related to cholesterol metabolism in livers. Gene involved in cholesterol elimination Cyp7a1 and Ldlr and cholesterol synthesis HMG-CoA reductase. Data are expressed as mean \pm SEM. $* \mathrm{P}<0.05$, $* * \mathrm{P}<0.01$ compared with $\mathrm{ND}$ group; $\# \mathrm{P}<0.05$, \#\#P $<0.01$ compared with $\mathrm{HCD}$ group.

Figure S5. Effects of riclin on the protein levels $(\mathrm{N}=3)$ related to cholesterol metabolism in intestines. 
Table S1. Primer sequences used for real-time PCR

\begin{tabular}{ccc}
\hline Gene & Forward primer sequences & Reverse primer sequences \\
\hline TNF $\alpha$ & 5'-ACGGCATGGATCTCAAAGAC-3' & 5'-CGGACTCCGCAAAGTCTAAG-3' \\
IL-6 & 5'-CATGTTCTCTGGGAAATCGTGG-3' & 5'-GTACTCCAGGTAGCTATGGTAC-3' \\
IFN- $\gamma$ & 5'-ATGAACGCTACACACTGCATC-3' & 5'-CCATCCTTTTGCCAGTTCCTC-3' \\
IL-1 $\beta$ & 5'-CATCCAGCTTCAAATCTCGCAG-3' & 5'-CACACACCAGCAGGTTATCATC-3' \\
NPC111 & 5'-GACATCACCTTCCACCTCTTG-3' & 5'-CTGGCATTCGACCCATGTAG-3' \\
Srb1 & 5'-ATGGGCCAGCGTGCTTTTATGAAC-3' & 5'-ACGCCCGTGAAGACAGTGAAGACC-3' \\
Abcg5 & 5'-AGCTCTTCCAACACTTCGAC-3' & 5'-TACGTTTCTATTTCCCGCTC-3' \\
Abcg8 & 5'-GACCTGGTCCTTCTGATGAC-3' & 5'-AGAGACTGTGCCTTCTCCAC-3' \\
Acat2 & 5'-AGACTTGGTGCAATGGACTCGAC-3' & 5'-CATAGGGCCCGATCCAACAG-3' \\
Mttp & 5'-TGAGCGGCTATACAAGCTCAC-3' & 5'-CTGGAAGATGCTCTTCTCGC-3' \\
Abca1 & 5'-TTGGCGCTCAACTTTTACGAA-3' & 5'-GAGCGAATGTCCTTCCCCA-3' \\
Srebp2 & 5'-CCAAGGAGAGCCTGTACTGC-3' & 5'-ACTGCTGGAGAATGGTGAGG-3' \\
Cyp7a1 & 5'-GCCTCTGAAGAAGTGAATGG-3' & 5'-TAAAAGTCAAAGGGTCTGGG-3' \\
Ldlr & 5'-TGACTCAGACGAACAAGGCTG-3' & 5'-ATCTAGGCAATCTCGGTCTCC-3' \\
Hmgcr & 5'-AGCTTGCCCGAATTGTATGTG-3' & 5'-TCTGTTGTGAACCATGTGACTTC-3' \\
$\beta$-actin & 5'-CCTGAACCCTAAGGCCAACC-3' & 5'-CAGCTGTGGTGGTGAAGCTG-3' \\
\hline
\end{tabular}

Table S2. Human equivalent doses of succinoglycan riclin

\begin{tabular}{|l|l|l|}
\hline C57BL $/ 6$ Mice doses & $2 \%$ diet & $4 \%$ diet \\
\hline Human equivalent doses & $0.24 \mathrm{~g} / \mathrm{kg}$ & $0.48 / \mathrm{kg}$ \\
\hline
\end{tabular}


A

A

HR

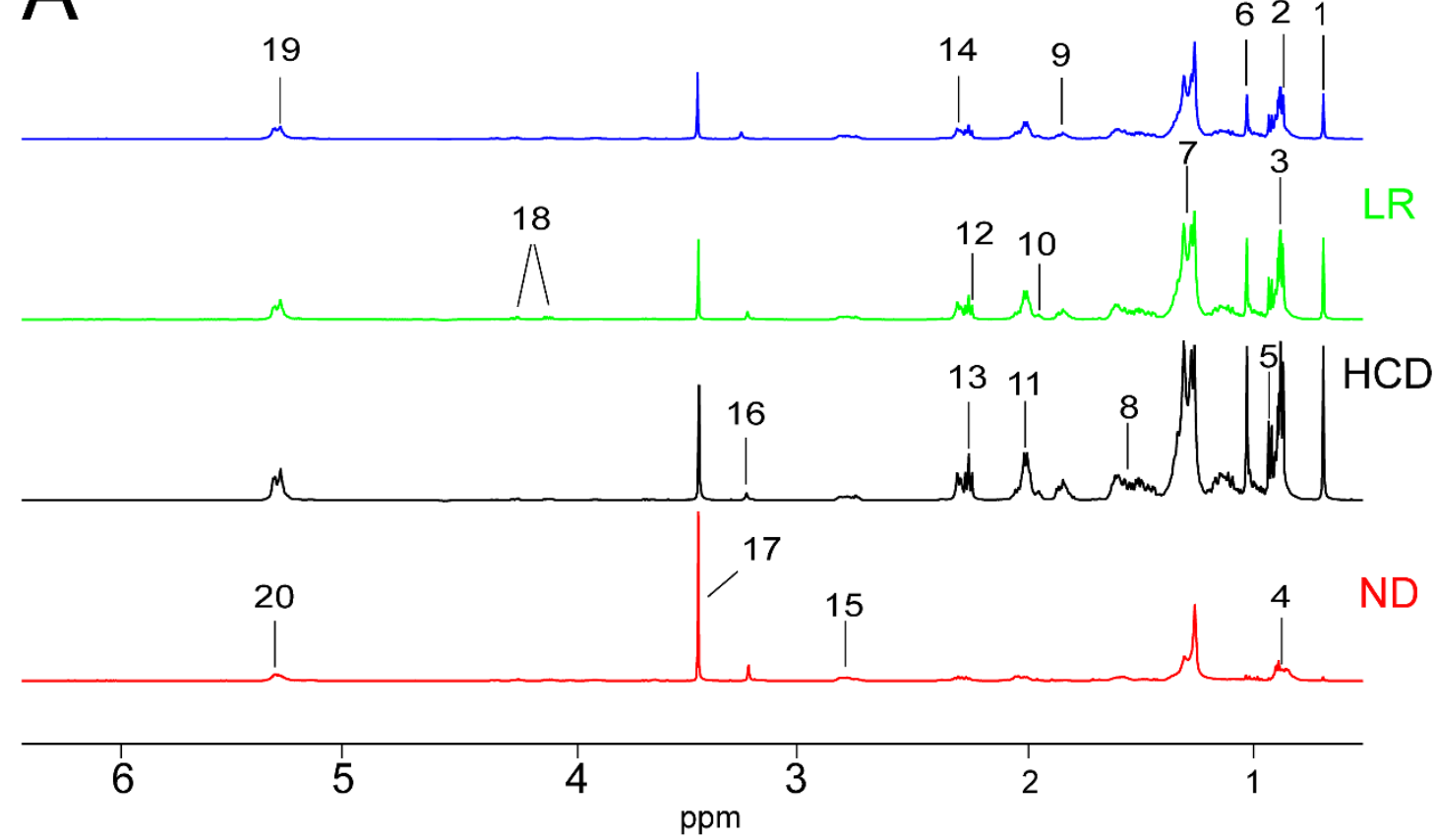

B
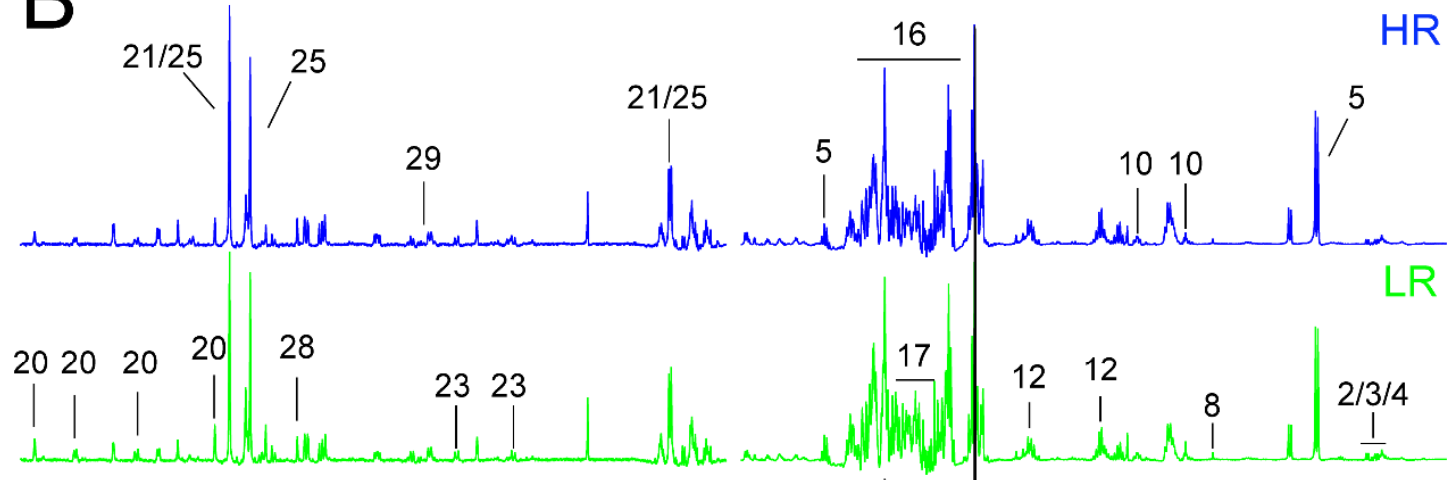

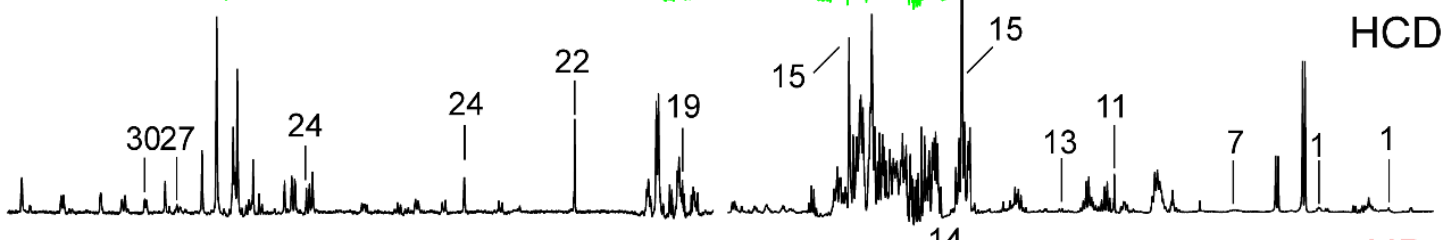

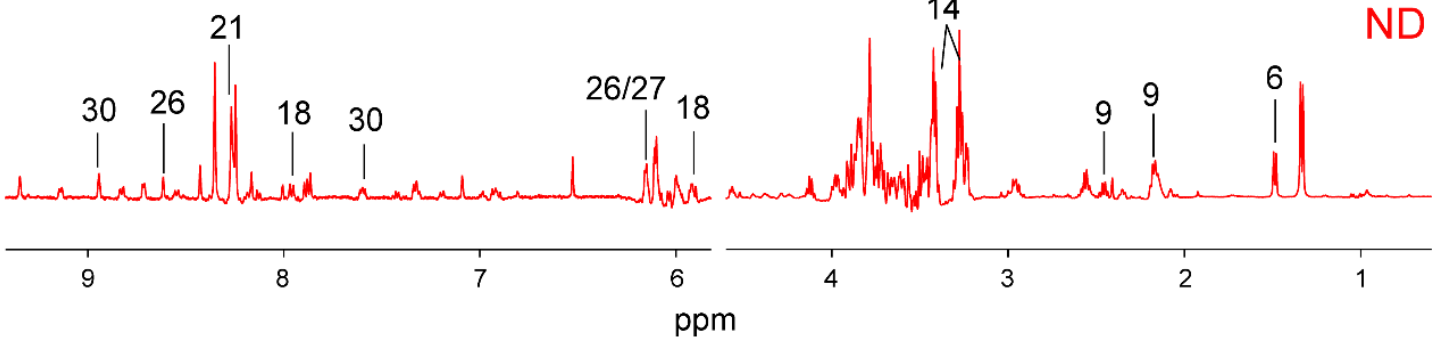

Figure S1 
A
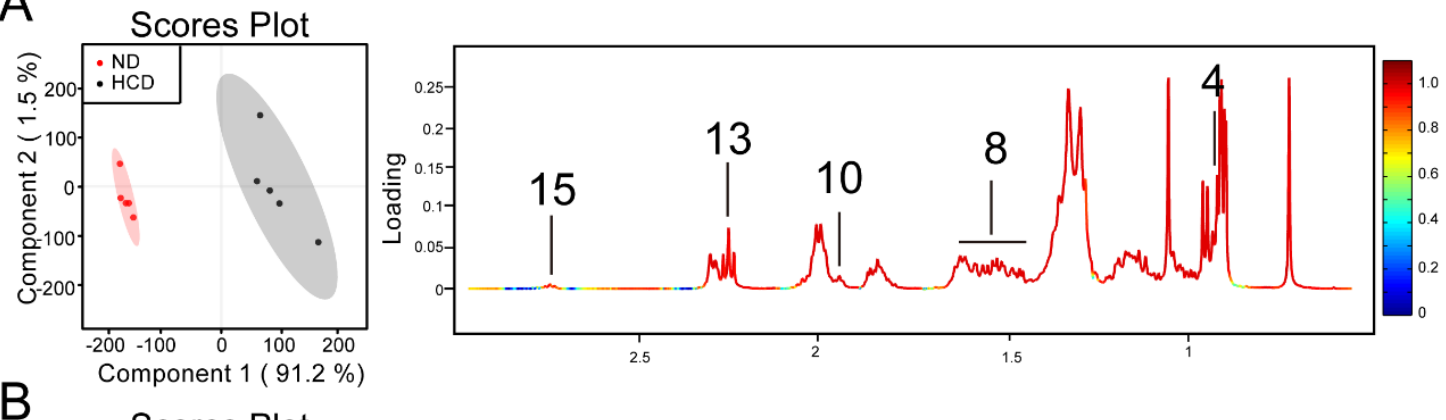

B
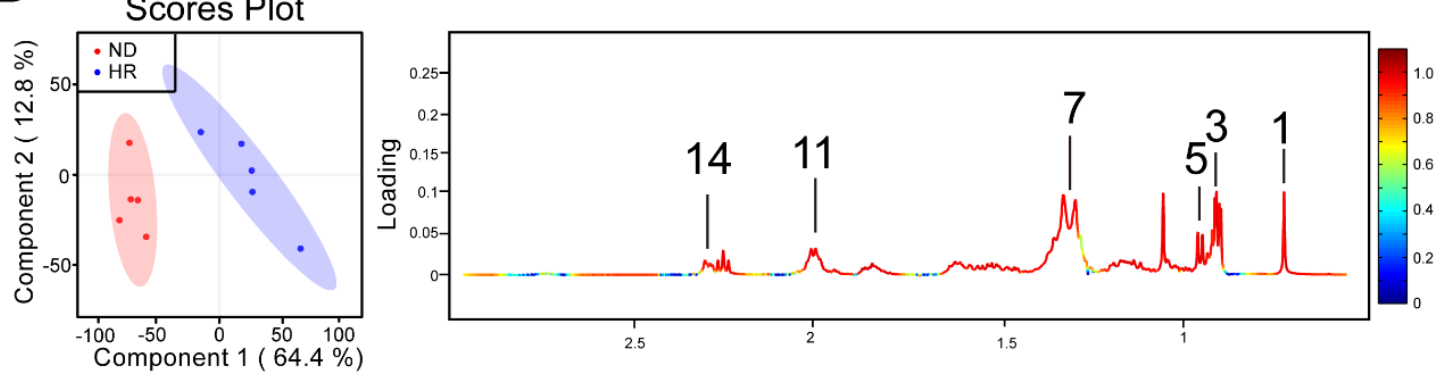

Figure S2 
A Scores Plot B
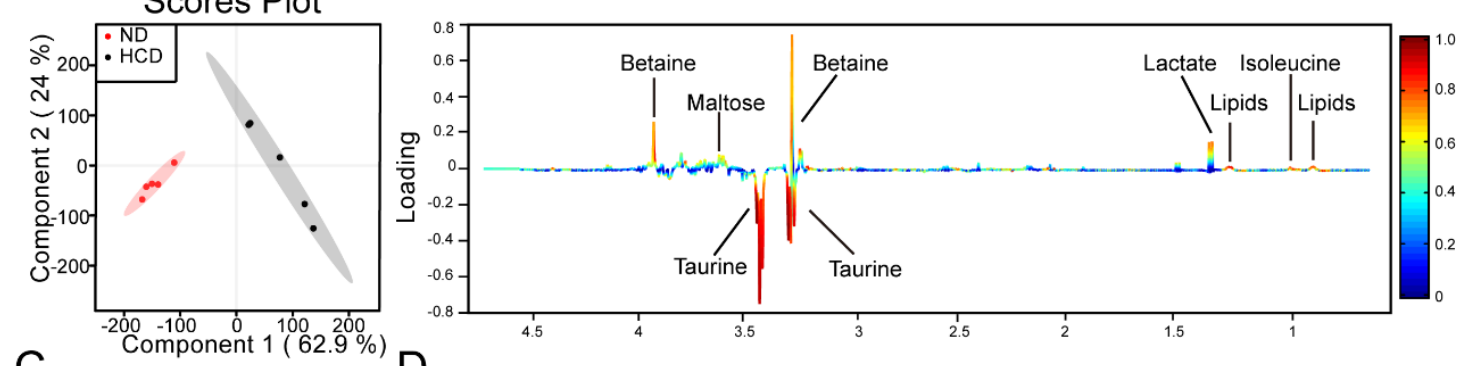

C S Plot D
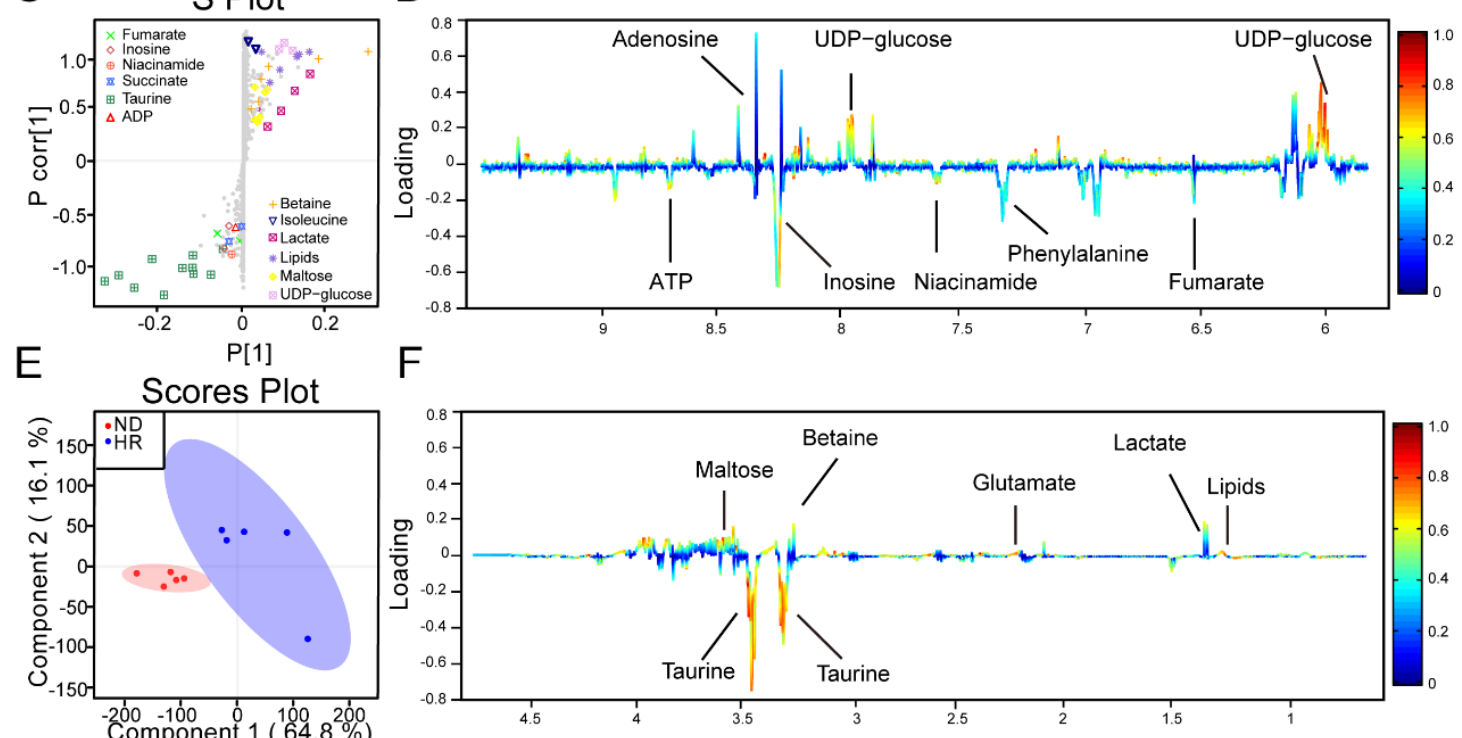

$\mathrm{F}$
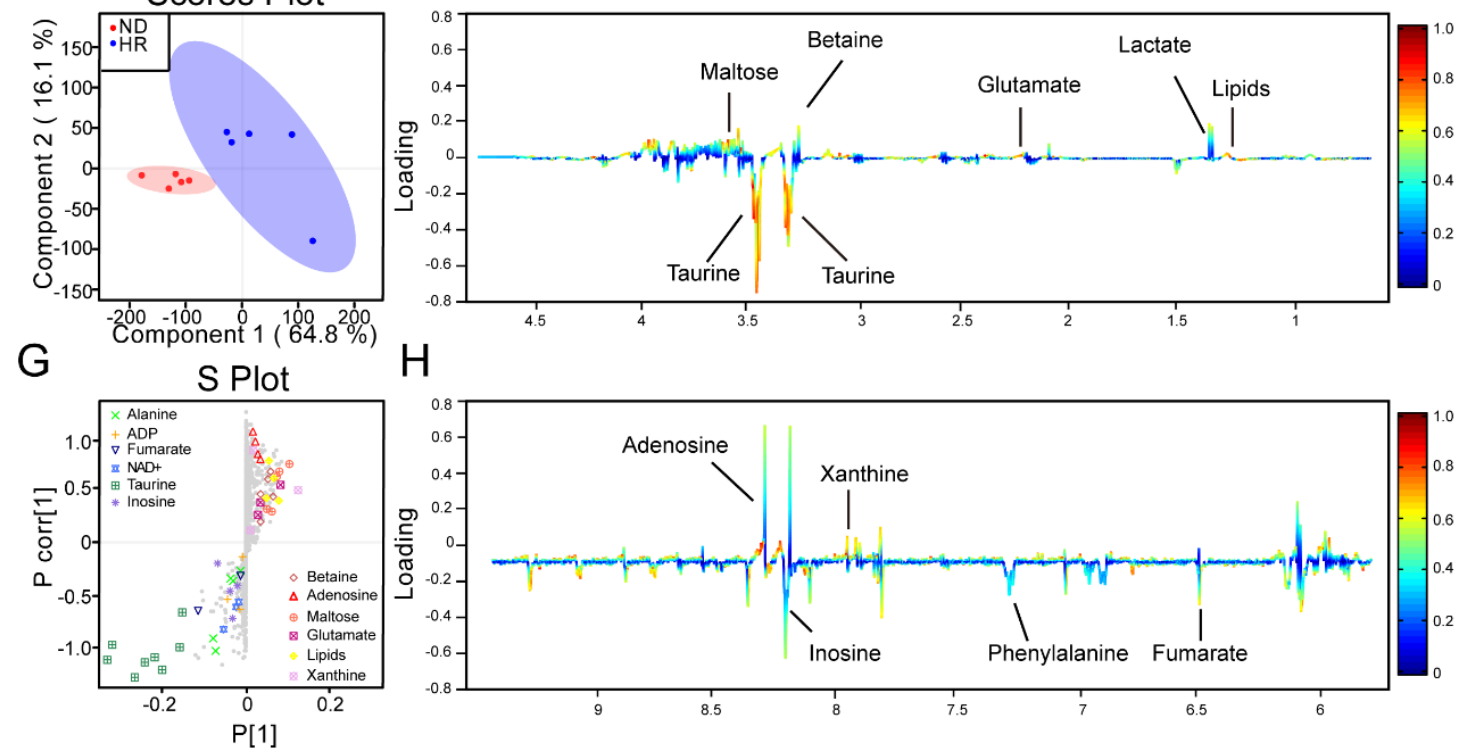

$\mathrm{H}$

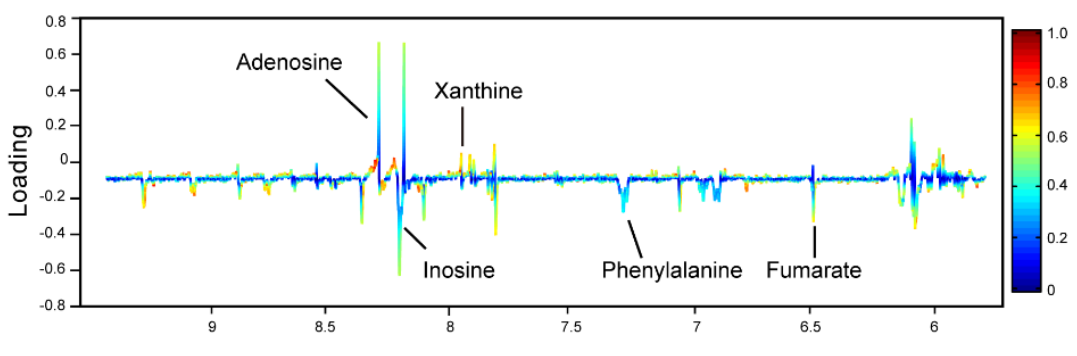

Figure S3 


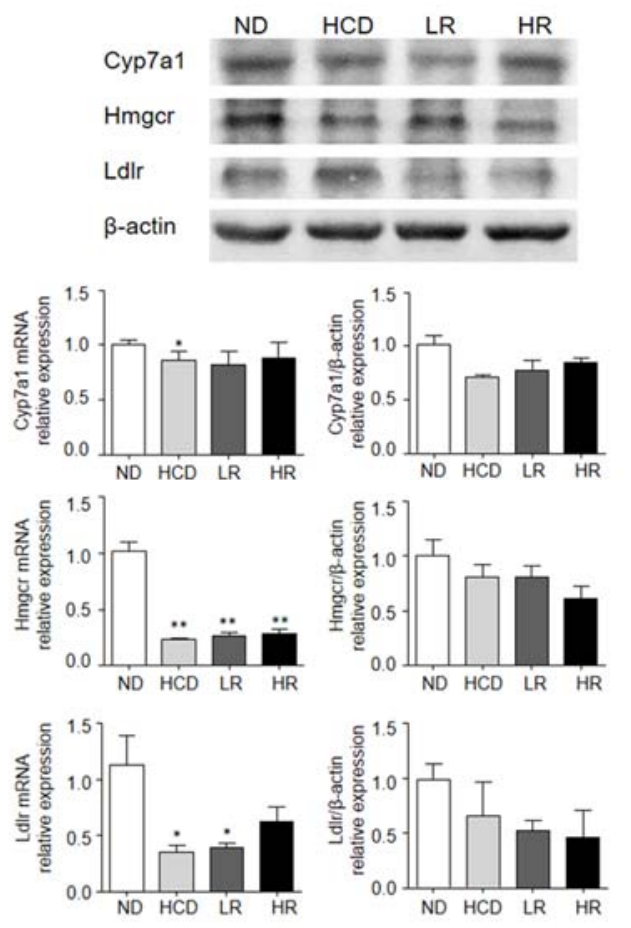

Figure S4 


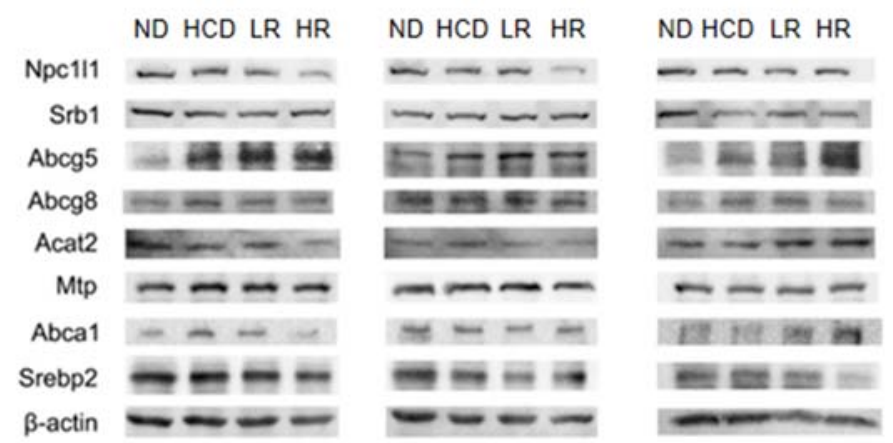

Figure S5 